\title{
La apuesta de Coca-Cola Life como marca icono: una mirada desde el branding cultural
}

\author{
Coca-Cola Life-n apustua ikur marka gisa:
} begirada bat kultur branding delakotik

Coca-Cola Life's bet as an iconic brand: A cultural branding perspective

\author{
Paloma Sanz-Marcos $\star$ \\ Universidad de Sevilla \\ Pablo Matus, Enrique Vergara \\ Pontificia Universidad Católica de Chile
}

RESUMEN: En 2013 se presentó en el mercado chileno Coca-Cola Life, una bebida carbonatada cuya principal característica es el uso de estevia como endulzante, lo que implica que tiene menos calorías y una composición más natural que la tradicional Coca-Cola. Sin embargo, y pese al auge del consumo verde - atento a los atributos ecológicos y sanitarios de los productos-, apenas dos años después de su lanzamiento las bajas ventas provocaron su retiro del mercado, situación que el producto ha vivido en otras latitudes. Desde un enfoque de branding cultural, la presente investigación describe las claves discursivas de la marca Coca-Cola Life para descubrir por qué no logró vincularse con los consumidores, pese a tener los atributos para ser considerada una «marca icono». Los resultados muestran que las campañas en Twitter en Chile (140 piezas entre 2013 y 2015) representaron de manera superficial las problemáticas e intereses ecologistas.

PALABRAS CLAVE: Branding cultural; publicidad verde; Twitter; Coca-Cola; medioambiente.

ABSTRACT: In 2013, Coca-Cola Life was introduced to the Chilean market. It is a carbonated beverage whose main characteristic is the use of stevia as a sweetener, which meant that it have fewer calories and a more natural composition than regular Coke. However, and despite the boom in green consumption-more attentive to the ecological and sanitary attributes of products-two years after its launch, low sales led to its withdrawal from the market... a situation that the product has lived in other latitudes. From a cultural branding approach, this study describes the discursive keys of the Coca-Cola Life brand to discover why it failed to connect with consumers, despite having the attributes to be considered an 'iconic brand.' The results show that the campaigns on Twitter in Chile (140 ads, between 2013 and 2015) superficially represented environmental issues and interests.

KEYWORDS: Cultural branding; green advertising; Twitter; Coca-Cola; environment.

\begin{abstract}
* Correspondencia a / Corresponding author: Paloma Sanz-Marcos. Departamento de Comunicación Audiovisual. Facultad de Comunicación. Universidad de Sevilla. Avda. Américo Vespucio, s/n (41092 Sevilla) - palomasanz@us.es - https://orcid.org/0000-0002-6103-6993

Cómo citar / How to cite: Sanz-Marcos, Paloma; Matus, Pablo; Vergara, Enrique (2020). «La apuesta de Coca-Cola Life como marca icono: una mirada desde el branding culturalı, Zer, 25(48), 85-103. (https://doi.org/10.1387/zer.21228).
\end{abstract}

Recibido: 07 noviembre, 2019; aceptado: 06 febrero, 2020.

ISSN 1137-1102 - elSSN 1989-631X / (C) 2020 UPV/EHU

(c) (i) Esta obra está bajo una licencia

Creative Commons Atribución 4.0 Internacional 


\section{Introducción}

A fines de 2013 The Coca-Cola Company presentó un nuevo producto destinado a renovar su oferta de bebidas carbonatadas y a satisfacer la emergente demanda por un consumo más saludable: Coca-Cola Life. No solo fue rupturista desde un punto de vista estético, pues en su etiqueta dominaba el color verde - a diferencia de las otras marcas de la empresa-, sino también desde su elaboración, ya que estaba endulzada con una mezcla de azúcar y hojas de estevia, una planta originaria de América del Sur, lo que en principio le permitía tener menos calorías que la versión original de Coca-Cola.

Pese a su carácter innovador y a sus atributos potencialmente positivos, así como a una modificación en su fórmula y sabor, no logró cautivar a los consumidores y en 2016 sus bajas ventas obligaron a la multinacional a retirar la bebida de diversos mercados (El Dínamo, 2016).

Tomando en cuenta las tendencias mundiales en torno al marketing sostenible y la vida sana, el fracaso de este producto es llamativo. Más todavía al considerar que fue respaldado por Coca-Cola, una de las principales marcas globales.

Asimismo, hay otro motivo por el cual es pertinente analizar este caso: CocaCola Life fue creada en Argentina y Chile - por iniciativa de profesionales de la compañía en ambos países-, y fue presentada al mundo desde estos mercados (Coca-Cola European Partners, 2014). Sin embargo, cuando se introdujo en Europa en 2014, en el Reino Unido surgieron una serie de cuestionamientos hacia su principal componente diferenciador, la estevia. De hecho, en aquella época ni siquiera en Estados Unidos había certeza de que efectivamente la bebida fuese más sana que la Coca-Cola original (Hofherr, 2014). Y aunque en la actualidad la FDA no considera peligrosos a los endulzantes elaborados con esta planta (US Food and Drug Administration, 2018), hay importantes mercados en los cuales la bebida no ha sido introducida por temor a las bajas ventas, como sucede en España (Valero, 2017).

Estas dudas sobre el producto han representado un impacto enorme para la marca. Desde el comienzo, Coca-Cola Life se publicitó como un producto más natural y más sano; por ello su estética - por ejemplo, en su logotipo y sus envasesadoptó el color verde (Truong, 2013). Sin embargo, la prensa inglesa cuestionó esto, considerando que una lata contenía 22 gramos de azúcar, un cuarto de la dosis diaria recomendada para un adulto (Wallop, 2014). Quizás por esto, desde diversos frentes se consideró a Coca-Cola Life como un mero esfuerzo de greenwashing por parte de la compañía (Wilson, 2013; Han, 2015).

Paradójicamente, en Argentina y Chile, así como en el Reino Unido, ya no se vende Coca-Cola Life debido a su baja aceptación por parte de los consumido- 
res (El Dínamo, 2016; Mazzuco, 2016). En España, en tanto, solo se vende en tiendas gourmet, aunque otra marca, Green Cola - una empresa griega que cuenta con concesionaria en la península-, también endulzada con estevia, está presente desde hace dos años y aparentemente ha tenido resultados positivos (Expansión, 2017).

Considerando estos antecedentes, el presente artículo indaga en la construcción discursiva y cultural de la marca Coca-Cola Life a partir del análisis de las piezas publicitarias distribuidas en Chile a través del medio social Twitter, entre 2013 (cuando se estrenó el producto) y 2015 (cuando la cuenta oficial de la marca dejó de postear mensajes).

\section{Marco teórico}

\subsection{LA MARCA DESDE UNA APROXIMACIÓN CULTURAL}

Actualmente las marcas han evolucionado hacia su consideración como discursos significativos para el consumidor más allá del producto. Así, la gestión de marca ha adquirido una mayor relevancia y sofisticación para los mercados (Ortegón, 2014), que cada vez manifiestan un mayor interés en la construcción de discursos que dialoguen con el universo cultural del consumidor (Fernández, 2013). El estudio de las marcas desde una perspectiva cultural ha sido reconocido desde un amplio ámbito disciplinar, donde convergen la antropología, la historia o la sociología, lo que otorga una gran complejidad conceptual a su formulación y desarrollo (Schroeder, 2009).

Esta operacionalización de la cultura por parte de la creación y el desarrollo de marcas ha sido abordada desde el enfoque del branding cultural (Heding, Knudtzen \& Bjerre, 2009) y, donde uno de sus principales exponentes es Douglas Holt. El modelo propuesto por este autor asume que la marca está inserta en una dinámica cultural, pues la base de su creación es la cultura en sí, lo que implica que los significados asociados a ella son colectivos. De este modo, el consumidor es visto como una pieza clave en el proceso del branding, y no solo como un destinatario de mensajes, ya que tanto el sujeto como su contexto son aspectos que participan de la creación de valor de la marca (Heding, Knudtzen \& Bjerre, 2009, p. 216). Esta perspectiva es muy diferente a otros modelos convencionales, debido a que concibe a la marca como un artefacto cultural en sí mismo (Holt, 2004).

Este modelo deviene de dos procesos: uno sustentado en la influencia de la cultura sobre la marca y otro basado en el impacto que esta tiene sobre el mercado y la cultura globalizada (Fernández, 2013, p. 135). Así, la marca se expresa como una narración de historias ancladas en diversos significados culturales, que al mismo tiempo operan como un factor importante en la intrincada red de sentido que dialoga con la identidad de los consumidores (Heding, Knudtzen \& Bjerre, 2009). 
Desde esta perspectiva, el producto es entendido por los consumidores como un conducto a través del cual ellos pueden experimentar las historias que la marca comunica. Luego, el valor del producto reside en su capacidad para proporcionar a los consumidores un modo de experimentar esas historias. El propio Holt (2004, p. 36) lo ilustra de esta manera: «Cuando los consumidores beben productos como Coca-Cola o Corona no están simplemente ingiriendo un líquido, sino experimentando 'mitos de identidad' a través de ese vínculo con la marca».

Vistas de este modo, las marcas se convierten en importantes agentes culturales. Lejos de entenderse como simples mediadores, serían más bien referentes ideológicos que ayudan a moldear la actividad económica, los rituales y las normas sociales a través de sus mitos (Vergara, 2006; Schroeder, 2009, p. 124; Porath, Vergara, Gómez-Lorenzini \& Labarca, 2017). Es más, las marcas no competirían realmente en un mercado de productos, sino en un «mercado de mitos» en el que los bienes/servicios ofrecen relatos capaces de orientar al consumidor y resolver incluso las contradicciones culturales que le preocupan y tendrían la capacidad de satisfacer necesidades sociales en un momento histórico determinado (Holt, 2004, p. 26).

\subsection{MARCAS ICÓNICAS Y TENSIONES SOCIALES}

Ollé \& Riu (2009, p. 112) señalan que cualquier cambio cultural, acontecimiento social o movimiento económico puede tener un impacto en el contexto de la marca y en los significados que se configuran en torno a la misma. En este escenario, son reconocidas como «marcas icónicas» aquellas que, legítima y efectivamente, representan ideas o mitos que se suponen - y se constituyen como- importantes para la sociedad. Bajo esta premisa, el principal propósito del branding cultural sería desarrollar el carácter icónico de una marca, pues esto le ayudaría a vincularse profundamente con los consumidores que se sientan identificados con dichos mitos (Fernández, 2013, p. 155).

Es por esto que la marca icónica es un sistema, pues posee un mecanismo que le permite absorber y reflejar la cultura que le rodea. Para Holt (2004, pp. 39-40) la marca es sensible a las tensiones y cambios sociales y por ello opera como un medio para llegar de manera más trascendente al consumidor.

Siguiendo esta perspectiva, el concepto de «icono cultural» define a símbolos que la gente ha aceptado como atajos para representar ideas importantes. Su esencia es que aquello que representan se considera como el símbolo más irresistible de un conjunto de valores o ideas que la sociedad aprecia como relevantes (Holt, 2004, pp. 183-184).

Batey (2013, p. 300) afirma que el primer sentido de lo icónico en nuestra cultura se encuentra en los iconos religiosos, cuyo valor se realzaba a través de los 
cuentos, las referencias en documentos sagrados y representaciones de lugares santos. Esto daría cuenta del carácter discursivo-narrativo del icono cultural, que del mismo modo caracterizaría hoy a las marcas icónicas.

No obstante, los mitos más influyentes serían aquellos que abordan - y ayudan a construir - las identidades de las personas. Siguiendo a Holt (2004), los consumidores utilizan marcas icónicas como bálsamos sociales: se apoyan en el mito mientras usan el producto como un medio para suavizar estas tensiones, ayudando a las personas a crear un propósito en sus vidas y a consolidar su identidad. En efecto, el mito identitario que representa la marca tendría la capacidad para relajar dichas tensiones. Eso explicaría que los individuos que adoptan el mito de la marca como insumo para sus propias identidades forjen conexiones emocionales tan estrechas con ella.

\subsection{Publicidad Verde y CONSUmidor Verde}

El concepto de publicidad verde [green advertising] surgió a principios de la década de 1990 para definir a aquellos mensajes que en algún grado establecen un vínculo entre el bien ofrecido y el entorno natural; que presentan una imagen corporativa de responsabilidad ambiental; que promueven un consumo asociado a un estilo de vida naturalista o ecologista, y/o que apelan a necesidades y expectativas del consumidor respecto de problemáticas ambientales (Banerjee, Gulas \& Iyer, 1995, p. 22; Zinkhan \& Carlson, 1995, p. 1).

En términos de marketing, este concepto se vincula estratégicamente con el de consumidor verde, aquel que más frecuentemente considera la conducta ambiental de las empresas y los efectos de sus productos o servicios en sus decisiones de compra (Iyer, Banerjee \& Gulas, 1994; Miller, 2009).

No obstante, la investigación sobre green advertising y su efecto en el consumidor verde no ha estado libre de controversias (Leonidou, Leonidou, Palihawadana \& Hultman, 2011, p. 9). Ya en los primeros estudios se descubrió que el consumidor verde era más crítico e incluso reacio a confiar en la publicidad (Carlson, Grove \& Kangun, 1993, p. 38; Shrum, McCarty \& Lowrey, 1995, p. 71; Zinkhan \& Carlson, 1995 , p. 5), sobre todo, cuando dichos mensajes eran evaluados como ambiguos, con información incompleta o falsa, fenómeno que, en términos críticos, fue entendido como un lavado de imagen ecológico o greenwashing (Kangun, Carlson \& Grove, 1991, pp. 54-55).

Uno de los primeros - y más citados - marcos conceptuales empleados para clasificar los mensajes publicitarios verdes es el propuesto por Carlson, Grove \& Kangun (1993, pp. 31-34). Para ellos es posible distinguir cuatro tipos de mensa- 
jes: los orientados a potenciar la imagen o la marca de la compañía o del producto, normalmente de manera declarativa; los orientados al producto y sus atributos, también de manera declarativa; los orientados al proceso de elaboración del producto, que destacan las gestiones de la empresa fabricante para potenciar su sostenibilidad, y los orientados a una problemática ambiental concreta a la cual el producto responde, cuyo mensaje posee una enunciación fáctica y a veces incluso técnica. La principal conclusión de estos autores es que los mensajes que tienden a orientarse a la marca y al producto suelen ser considerados más vagos y ambiguos.

Algunos estudios recientes confirman que el consumidor verde tiende a dudar de la publicidad (Do Paço \& Reis, 2012, p. 153), sobre todo de aquella considerada dudosa o ambigua. Sin embargo, esto no ocurre con la publicidad más directa e informativa positiva (Matthes \& Wonneberger, 2014, p. 125).

En este sentido, se ha postulado que una mayor conciencia ambiental motiva un mayor interés y una mayor receptividad ante los mensajes de temática o connotación ecológica, sin importar el tipo de argumento o de anuncio (Tucker, Rifon, Lee \& Reece, 2012, p. 17). Esto se explicaría por la existencia de programas de marketing más concretos y efectivos en su propuesta ambiental, cada vez más basada en valores sociales - y ya no tanto en los productos y sus atributos-, lo que ha generado una publicidad verde más informativa (Leonidou, Leonidou \& Kvasova, 2010; Ahern, Bortree \& Smith, 2013; Matthes \& Wonneberger, 2014).

Con todo lo anterior, y pese a la relevancia de Coca-Cola como marca global, casi no existen estudios sobre el caso de Coca-Cola Life, el desarrollo de su marca y su publicidad. Apenas un par de publicaciones mencionan al producto (Spessot, Testa, Van Kooten, Velásquez, Villalón \& Saravi, 2016; Regatto \& Viteri, 2017; Crass, Czarnitzki \& Toole, 2019), pero siempre de manera tangencial y sin indagar en los tópicos que aborda este artículo.

\section{Metodología}

Considerando la inexistencia de publicaciones académicas respecto al caso de estudio, esta investigación posee un carácter exploratorio. Su aproximación teórica al objeto de estudio se ha orientado desde la perspectiva del branding cultural, por lo que su abordaje ha sido de carácter interpretativo y crítico. Lo anterior ha supuesto un trabajo en dos etapas: un análisis temático de tipo cuantitativo aplicado al corpus de piezas identificadas, y un análisis semiótico del discurso aplicado a una submuestra representativa de los grandes tópicos identificados.

El análisis temático corresponde a la identificación de categorías tópicas que permiten organizar un conjunto de casos a partir de orientaciones teóricas relativas 
al objeto de estudio (Bryman, 2012, pp. 578-581). En otras palabras, se trata de reconocer los asuntos o issues de los textos que constituyen el total de piezas identificadas. A diferencia del clásico análisis de contenido, basado en la observación de características explícitas del texto - por ejemplo, la cantidad de veces que se usa una palabra- (Neuendorf, 2002, p. 1), el análisis temático es el resultado de una interpretación subjetiva pero sistemática del mismo.

En el caso del análisis del discurso se han tomado como referentes los trabajos de orientación semiótica de Barthes (1986 [1964]), Greimas \& Courtés (1990), Williamson (1978), Abril (1995) y Bernárdez (2007), a partir de los cuales la observación se entiende como un ejercicio interpretativo respecto de los procesos de significación y sentido, caracterizados por una aproximación estructural al texto y capaz de profundizar en aspectos valóricos e ideológicos. De ahí que este enfoque permita mirar más allá del texto, reconociendo incluso aspectos contextuales y culturales, en cuanto a sistema de creencias y prácticas de valor simbólico (Geertz, 1990, p. 88).

Mediante ambos análisis se ha buscado identificar la orientación argumental de los mensajes, así como advertir de los aspectos culturales que operaron como anclajes narrativos en las piezas.

Coca-Cola Life fue lanzada al mercado chileno en octubre de 2013 y sus campañas publicitarias se difundieron en televisión, radio, prensa, vía pública, web y medios sociales. Pensando en abarcar la mayor cantidad y diversidad de piezas, la muestra se ha definido de manera censal a partir de todos los mensajes difundidos en la cuenta en Twitter de la marca (todavía disponible ${ }^{1}$ ) entre 2013, cuando se presentó el producto, y 2015, cuando se dejaron de postear mensajes. En total, fueron identificadas y recolectadas 140 piezas.

Es importante advertir que las piezas no necesariamente poseen la clásica estructura temática y discursiva de la publicidad — la combinación de mensaje visual y verbal (Barthes, 1986), y por tanto la existencia de una promesa u oferta expresada un eslogan (Russell, Lane \& King, 2005, pp. 484-493)—, pues consisten en imágenes que, de manera figurativa, asocian el producto y/o la marca con diversas escenas cotidianas. Sin embargo, esta es una práctica normal en medios sociales, pues se estima que de este modo, al no parecer abiertamente un aviso publicitario, el texto motivará más interacciones (likes, retweets) entre los usuarios (Mangold \& Faulds, 2009, pp. 363-364; Jolly, 2019).

\footnotetext{
1 En la web: https://twitter.com/cocacolaLife_cl.
} 


\section{Resultados}

\subsection{AnÁlisis temático}

De acuerdo con lo expuesto en el marco teórico, dos formas de categorización parecieron las más adecuadas para el análisis temático de los mensajes en Twitter.

La primera fue la formulada por Carlson, Grove \& Kangun (1993, pp. 31-34) respecto de las orientaciones argumentales de la publicidad verde. En este caso, se buscó determinar si los mensajes se centraban en el producto, en su imagen/marca, en la sostenibilidad de su proceso de fabricación o en una problemática ambiental.

La segunda es la propuesta por Heding, Knudtzen \& Bjerre (2009) respecto de la construcción de la marca mediante historias ancladas en significados culturales. En este caso se identificó el tipo de actividad o práctica cultural promovida en las piezas publicitarias. Este ejercicio se realizó primero de manera espontánea (identificamos la o las acciones representadas; p.e. caminar en un bosque) y luego de manera sintética, agrupando acciones similares y formando clusters temáticos (p.e. vivir la naturaleza).

Por tanto, mientras que en el primer análisis se emplearon categorías a priori, en el segundo se utilizaron categorías emergentes.

Como se ha advertido, los posts recolectados no necesariamente poseen un eslogan, lo que en cierto modo dificulta la interpretación de su mensaje. Sin embargo, precisamente por ser parte de mensajes en Twitter, todas las piezas se publicaron acompañadas de un enunciado verbal que permitió orientar su lectura.

La lectura de las imágenes se concentró en las dimensiones de referencia y figurativización, es decir, de representación de aspectos concretos y abstractos de la realidad, respectivamente (Greimas \& Courtés, 1990). A partir de esto se ha podido interpretar que un post se centraba en el producto y sus atributos cuando este aparecía referencialmente (p.e. se muestra la botella); del mismo modo, fue posible reconocer de manera figurativa un énfasis en la marca cuando, junto a una situación cualquiera (p.e. una mujer camina junto a su perro en el campo), solo se apreciaba el logotipo.

La tabla 1 muestra los resultados del análisis temático desde el punto de vista de las orientaciones argumentales propias de la publicidad verde y su relación con las actividades o prácticas culturales representadas en los mensajes. En ella se aprecia una clara orientación de las piezas hacia un discurso anclado en la imagen de marca de Coca-Cola Life y sus valores (65\%), el cual más que duplica al centrado en el producto y sus atributos $(29,29 \%)$, que ocupa el segundo lugar. 
TABLA 1

Análisis temático de los mensajes en Twitter $(\mathrm{N}=140)$

\begin{tabular}{lrr}
\hline Orientación argumental verde / Actividad representada & $\mathbf{n}$ & \% del total \\
\hline Imagen o marca & 91 & 65,00 \\
Compartir con otros (no familia) & 26 & 18,57 \\
Vivir (en) la naturaleza & 23 & 16,43 \\
Relaciones familiares & 18 & 12,86 \\
Disfrutar el tiempo libre & 14 & 10,00 \\
Ser optimistas/felices & 4 & 2,86 \\
Vida sana y/o más natural & 3 & 2,14 \\
Ser atrevidos/creativos & 3 & 2,14 \\
\hline Producto y sus atributos & 41 & 29,29 \\
Consumir el producto & 38 & 27,14 \\
Vivir (en) la naturaleza & 2 & 1,43 \\
Compartir con otros (no familia) & 1 & 0,71 \\
\hline Sostenibilidad de la fabricación & 6 & 4,29 \\
Consumir el producto & 5 & 3,57 \\
Compartir con otros (no familia) & 1 & 0,71 \\
\hline Problemática ambiental & 2 & 1,43 \\
Vida sana y/o más natural & 1 & 0,71 \\
Protegerse del sol & 1 & 0,71 \\
\hline Fuente:
\end{tabular}

Fuente: elaboración propia.

En las piezas cuyo argumento se ancla en la marca y sus valores se advierte una interesante dicotomía entre la promoción de prácticas que podrían considerarse hedonistas — orientadas a la búsqueda de placer o beneficio individual—, como «Vivir (en) la naturaleza», «Disfrutar el tiempo libre», «Ser optimistas/felices», «Vida sana y/o más natural», y «Ser atrevidos/creativos» (juntas suman un 33,57\% del total), y narrativas basadas en actividades más bien colectivas-emotivas, como «Compartir con otros»y, sobre todo, la apelación a «Relaciones familiares» (31,43\% del total). Puede decirse que esta dualidad constituye la primera clave discursiva de la publicidad en Twitter de Coca-Cola Life en Chile.

En el caso de la publicidad cuyo eje argumental es el producto y sus atributos, la apelación al consumo individual del mismo es la más frecuente $(92,68 \%$ de este grupo; $27,14 \%$ del total), lo que dialoga con la lógica hedonista advertida en el grupo anterior. El consumo del producto bien podría haber sido representado en el contexto del «Compartir con otros», no obstante, se apeló fundamentalmente a invitaciones individuales.

Un aspecto por destacar de la distribución temática de la muestra es la baja apelación al proceso de fabricación del producto $(4,29 \%)$, considerando que uno de los 
atributos clave de Coca-Cola Life es su fórmula. Esto se explica debido a que la inclusión de estevia en la bebida no tiene ningún efecto ecológico, sino solo un eventual beneficio sanitario (menos calorías).

En ese sentido, la escasa mención de problemáticas ambientales $(1,43 \%)$ sugiere que, pese a la frecuente alusión a la naturaleza, las campañas de esta marca —al menos en Twitter - no necesariamente corresponden a una publicidad verde es un sentido estricto.

\subsection{ANÁlisis DEL DisCURSO}

Un primer nivel de observación discursiva tuvo como objeto de estudio la construcción gráfica de la marca Coca-Cola Life. En el caso del logotipo, aunque este remite al estilo tradicional de la marca, la incorporación de la palabra «Life» agrega elementos nuevos. Por ejemplo, se emplea una tipografia gestual que, sin ser idéntica a la tradicional, dialoga con sus formas tradicionales, lo que permite entender que se trata de una marca emparentada con la original. No obstante, la gestualidad representada en la palabra "Life» — el hecho de que parezca escritura a mano alzada - implica una espontaneidad y naturalidad que el logotipo histórico, mucho más elaborado y con formas más cuidadas, no posee. Esto también la diferencia de las otras marcas de la compañía, Coca-Cola Light (igual de elaborada) y Coca-Cola Zero (más simple y moderna).

El uso del color verde como fondo para el logotipo es un factor diferenciador respecto de las otras marcas y potencia el vínculo de la marca Life con el discurso social sobre lo natural.

Otro nivel de análisis es la representación material del producto. En todos los casos donde aparece en las piezas, el display utilizado es la botella de vidrio individual, con el clásico diseño de Coca-Cola. En ella, tanto la etiqueta como la tapa reproducen la gráfica del logotipo. Lo llamativo del uso de la botella es su significado como opción de consumo personal - no grupal ni familiar- y el valor que ha recuperado el vidrio como material reciclable y natural, a diferencia de los envases de lata y plástico.

Desde este punto de vista, el display utilizado en los posts dialoga con los anclajes discursivos identificados en la marca. Hay que señalar, sin embargo, que este protagonismo de la botella de vidrio solo se advirtió a nivel de piezas publicitarias, pues en lo que respecta a puntos de venta Coca-Cola Life circuló en diversos formatos, materiales y tamaños.

Considerando que el mayor porcentaje de mensajes en Twitter (65\%) se orientó argumentalmente a la categoría «Imagen o marca» (tabla 1) y que el enfoque 
teórico adoptado (branding cultural) de hecho privilegia esta dimensión en el estudio de casos, en este artículo solo se presentan los resultados del análisis del discurso aplicado a esta categoría.

Para ejemplificar el análisis se seleccionaron de manera intencional tres piezas correspondientes a las subcategorías más recurrentes: «Compartir con otros», "Vivir la naturaleza» y «Relaciones familiares». Estas piezas fueron elegidas por su riqueza simbólica y su capacidad para representar a sus respectivos clusters, respondiendo a la lógica interpretativa de esta etapa del análisis. En consecuencia, no se persiguió una representación estadística de los resultados, sino solo ilustrar las claves de cada uno de los grupos.

En el caso de la subcategoría «Compartir con otros» - mensajes que representan actividades de tipo social o que implican el disfrute del tiempo con otros-, las piezas muestran acciones situadas en exteriores naturales, entendiendo por tales espacios con la menor intervención posible del ser humano. Para Coca-Cola Life, entonces, la verdadera naturaleza no se manifiesta en el parque o en el jardín, sino principalmente en el campo y el bosque. Es, en el fondo, una invitación a vivir juntos al aire libre.

En estos mensajes se sugiere un vínculo entre el entorno natural y la relación existente entre los personajes, lo que implica que el contexto es, en cierto modo, parte de dicha relación. Por ejemplo, en la figura 1, se muestra a una mujer que admira un paisaje junto a su perro; dado que este a su vez también forma parte de «lo natural» - lo no humano y no artificial-, es posible inferir que la relación entre ella y la naturaleza es más que solo contemplativa. El consumidor de Coca-Cola Life, por lo tanto, abraza a la naturaleza, a lo natural.

En el caso de la subcategoría "Vivir (en) la naturaleza» — mensajes que representan actividades individuales de goce o disfrute de la vida al aire libre- es destacable el hecho de que todos los sujetos representados son mujeres, lo que en cierto modo responde al imaginario naturalista de lo femenino, manifestado, por ejemplo, en la figura mítica de la «madre naturaleza» y en la asociación de la belleza femenina con la belleza natural. Esto adquiere más valor cuando se toma en cuenta que en ninguna de estas piezas hay grupos o parejas: para la marca, la belleza natural es algo que se experimenta o se vive de manera subjetiva e intimista.

Ejemplo de este grupo de mensajes es el de la Figura 2, en la que se muestra a una mujer joven, vestida de manera casual que juega sobre un columpio y disfruta del momento, rodeada solo por árboles propios de la geografia central del país (arrayanes). No se implica la presencia de alguien más, ni parece haber un contexto social que justifique la presencia de la mujer, y en ese marco el llamado («Ejercicios para el alma») potencia el carácter intimista de la escena. Ella disfruta por sí misma; solo 
le basta el contacto con la naturaleza. La fotografia, en perspectiva contrapicada, no solo permite ver con claridad los árboles y el cielo, integrados a la mujer en el cuadro, sino que remite a un estado superior de felicidad.

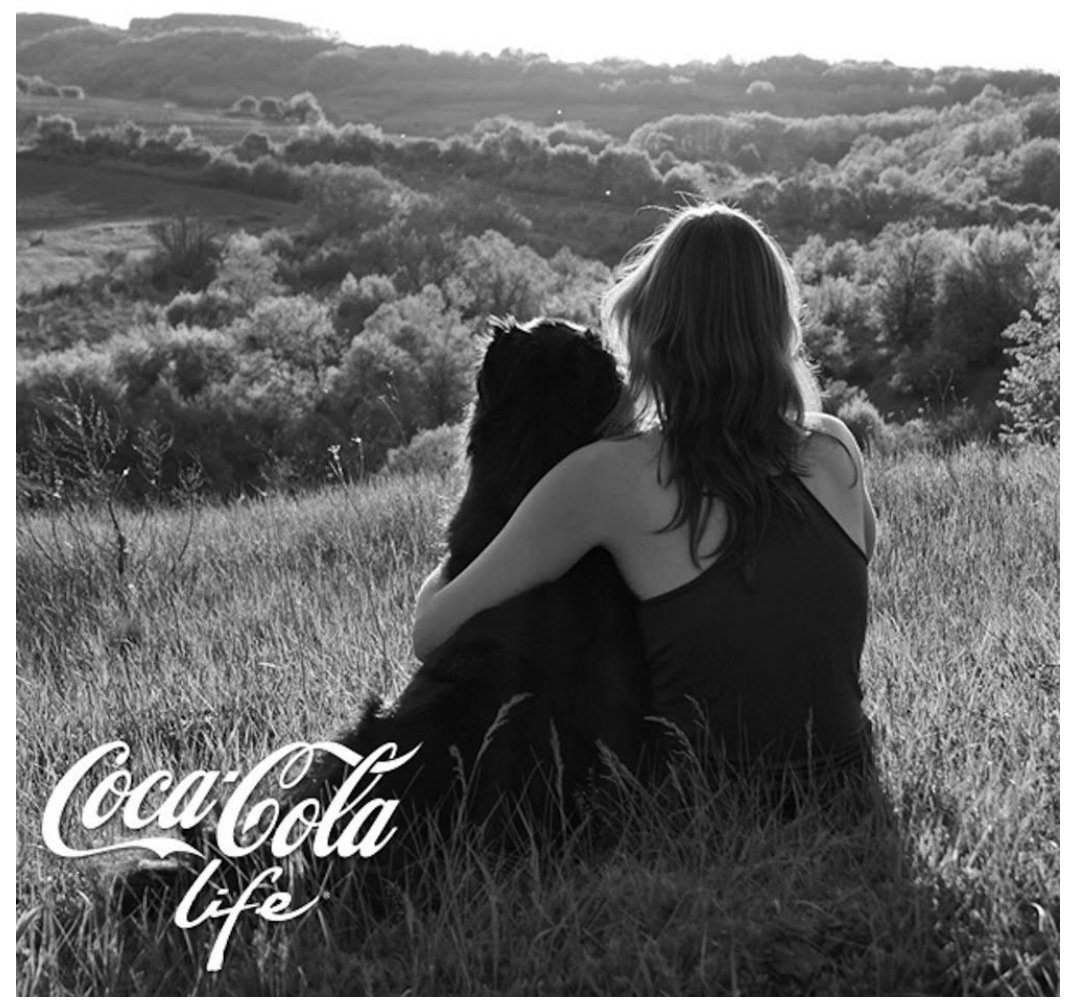

Fuente:@CocaColaLife_cl (Twitter); publicado el 23 de enero de 2014.

FigURA 1

Mujer abraza a su perro mientras ambos contemplan un paisaje (mensaje de la categoría "Compartir con otros»)

En los casos de esta subcategoría — así como en la imagen de ejemplo- no se hace referencia al producto ni a su consumo. En ese sentido, la gratificación del consumidor está dada por su vivencia personal y por su contacto con la naturaleza. De ahí que la presencia del logotipo de Coca-Cola Life se entienda como un recurso retórico para aludir a la adopción de la escena representada por parte del universo simbólico de la marca. Esto se reafirma a través del mensaje de la Figura 2, que apela a «Ejercicios para el alma», lo que implica, a diferencia de la subcategoría anterior, que lo importante no es verse bien, sino lograr un estado de plenitud trascendente e integral. 


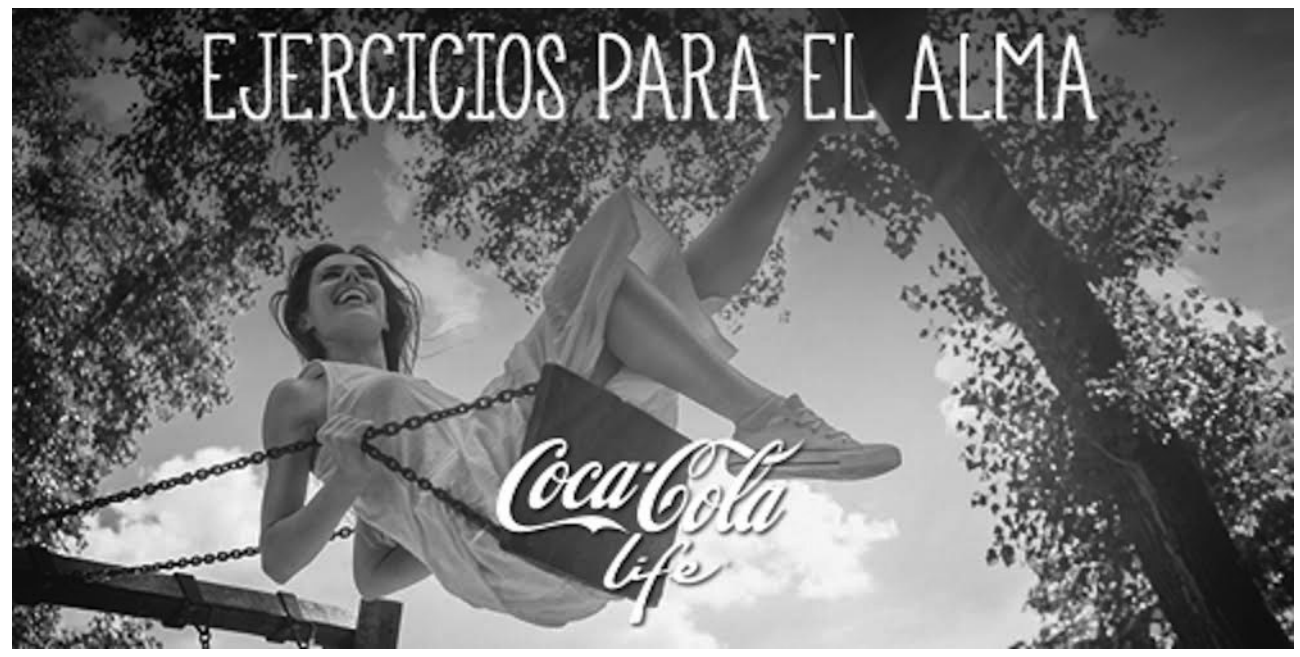

Fuente: @CocaColaLife_cl (Twitter); publicado el 10 de abril de 2015.

Figura 2

Mujer disfruta sobre un columpio, rodeada por árboles (mensaje de la categoría «Vivir [en] la naturaleza»)

Finalmente, en el caso de la subcategoría «Relaciones familiares» — mensajes que representan y promueven vínculos filiales y parentales-, probablemente lo más interesante es que la mayoría de las piezas ilustra a la familia mediante vínculos padre-hijo(a), lo que no solo constituye un cambio respecto de los estereotipos clásicos sobre el tema, que tienden a destacar el rol de madre como eje del núcleo familiar, sino que además plantea un encuadre distinto para el universo simbólico de la marca, hasta ahora muy vinculado a lo femenino. Esto podría entenderse como un esfuerzo por dialogar con los cambios culturales respecto al rol del hombre, de quienes ya no se espera que sea proveedor material de su familia, sino también agente activo en la crianza, y que no teman expresar sus sentimientos.

Un ejemplo de este enfoque es la Figura 3, donde se aprecia a un hombre adulto-joven que lleva en sus hombros a una niña, presumiblemente su hija, mientras sonríe. Es claramente un gesto afectivo y de complicidad. Por ello destaca la presencia explícita del producto: el hombre lleva en su mano izquierda una botella de Coca-Cola Life. En cierto modo, la marca se hace parte de la relación y de ese cariño. 


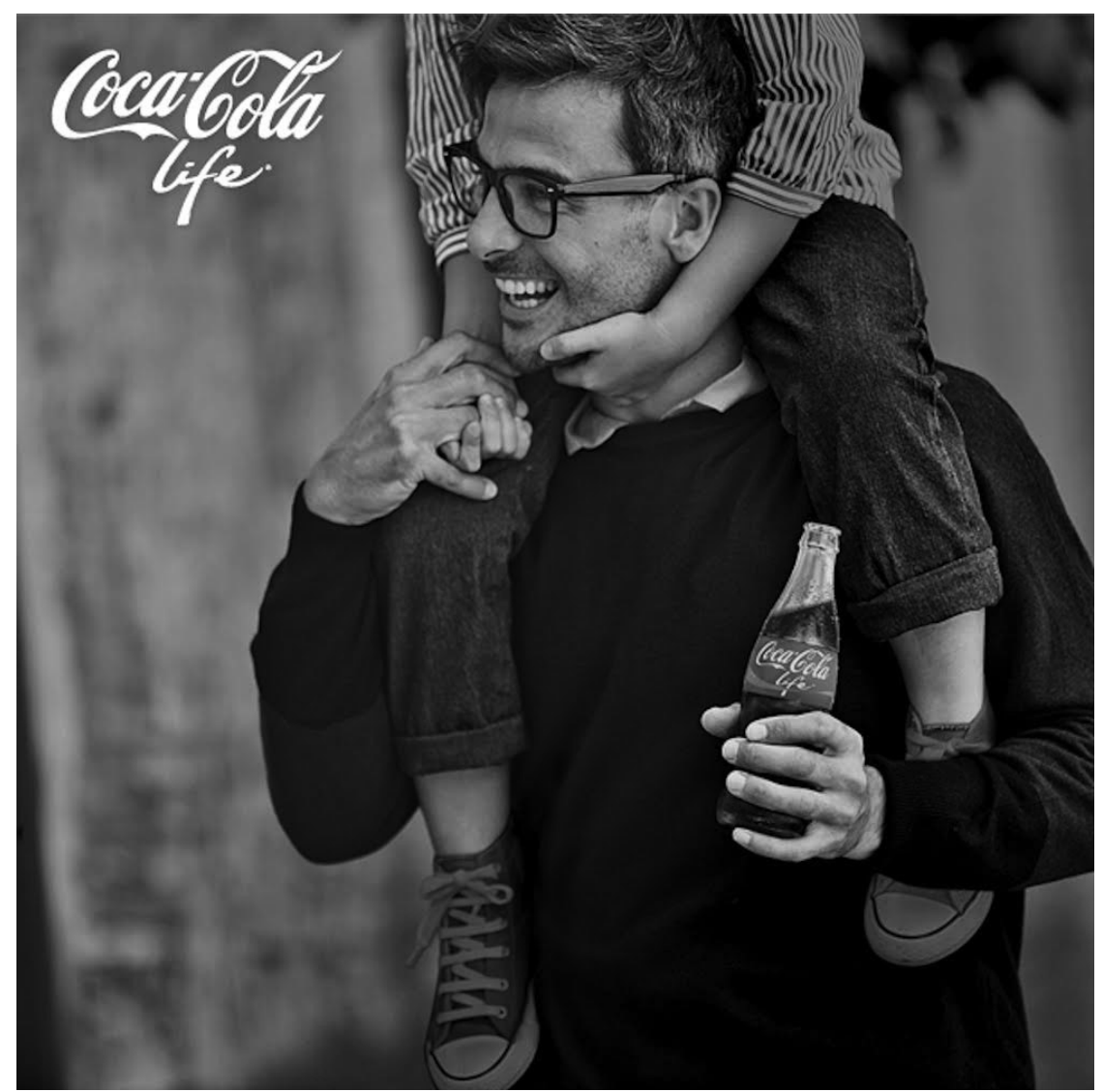

Fuente:@CocaColaLife_cl (Twitter); publicado el 25 de diciembre de 2013.

Figura 3

Hombre camina con su hija en los hombros y una Coca-Cola Life en la mano (mensaje de la categoría «Relaciones familiares»)

\section{Conclusiones}

Si bien la publicidad de Coca-Cola Life analizada puede considerarse dentro de la categoría de green advertising, lo cierto es que en su enorme mayoría no posee un mensaje con vocación ambientalista, sino más bien presenta lugares comunes y alusiones superficiales a la naturaleza (paisajes, personas que disfrutan del entorno natural). Esto puede explicarse por las condiciones del producto, cuyo gran atributo - el uso de estevia como endulzante- no responde a una problemática ecológica, sino más bien a un interés sanitario y hedonista. 
Desde la perspectiva de un consumidor verde, probablemente Coca-Cola Life no se presenta como una marca con un carácter plenamente ecológico, algo que ha podido influir en la escasa aceptación del producto, al menos por este tipo de consumidores. En cuanto al consumidor tradicional, la situación no necesariamente ha sido mejor. Coca-Cola Life apeló a su carácter más saludable, dada su composición en base a estevia, pero esto solo reducía las calorías de la bebida en comparación a la versión original, no las eliminaba. Esto explica la segunda etapa de la campaña donde se apeló a una nueva formulación. Si se considera que tanto Coca-Cola Light como Coca-Cola Zero resultan más efectivas en ese sentido, es posible advertir que la propia competencia interna afectó también a las posibilidades de éxito de la versión Life.

Otro aspecto potencialmente conflictivo de estas campañas es la disonancia generada al presentar un producto con asociaciones ecológicas, o al menos naturalistas, comercializado en displays regulares (latas y plásticos), que no necesariamente satisfacen la preocupación de un consumidor verde. Es cierto que en la publicidad analizada solo se exhibían botellas de vidrio, pero en los puntos de venta la experiencia del potencial consumidor era diferente. En este contexto podría sugerirse que, a juicio de un consumidor verde, la publicidad de Coca-Cola Life tuvo connotaciones de greenwashing.

Esta idea se relaciona con la perspectiva del branding cultural. Los análisis aplicados a las piezas publicitarias permiten sostener que Coca-Cola Life pudo haberse constituido como una marca icónica, pues su discurso se construyó apelando a issues propios del contexto actual — como el interés por una vida más sana y natural, y el deseo de cuidar el planeta- tanto como a aspiraciones tradicionales y universales - p.e. el placer de compartir con otros y de experimentar una familia-, y en ese sentido buscó aliviar ciertas tensiones sociales. En definitiva, la marca recurre a diversos y profundos mitos.

Asimismo, son interesantes los encuadres adoptados para representar roles de género en la campaña, los cuales dialogan con una serie de movimientos ciudadanos contemporáneos y cambios culturales que ha experimentado el país (Gómez-Lorenzini, Vergara, Labarca \& Porath, 2016; Vergara \& Porath, 2017; Porath, Vergara, Gómez-Lorenzini \& Labarca, 2017). No solo las mujeres representadas en las piezas son individuos activos y plenos por sí mismos; los hombres, en el contexto de la familia — con hijos_-, son sujetos maduros emocionalmente, que no temen expresar sus sentimientos y que asumen felices un rol más completo que el de mero proveedor material.

Estas características discursivas de la publicidad de Coca-Cola Life hacen de este caso algo muy llamativo. Siguiendo la teoría, al ser una marca construida desde el diálogo con narrativas culturales tradicionales y contemporáneas, y con la capacidad 
para apropiarse de imágenes significativas desde el punto de vista valórico, su identidad y su discurso claramente representaban importantes características de la sociedad actual. Sin embargo, desde un punto de vista comercial el producto fue un fracaso en todas partes. Esto quiere decir que los anclajes culturales de esta marca icónica no funcionaron adecuadamente, al menos desde una perspectiva discursiva.

Futuras investigaciones podrán profundizar en esta reflexión, sobre todo al considerar otras dimensiones de análisis como, por ejemplo, la experiencia de los directores ejecutivos y creativos responsables de la campaña o la evaluación de esta por parte de los propios consumidores, lo cual podría complementar y enriquecer los hallazgos y conclusiones expuestos en este artículo.

\section{Referencias bibliográficas}

Abril, G. (1995). Análisis semiótico del discurso. En J.M. Delgado \& R. Gutiérrez (eds.), Métodos y técnicas cualitativas de investigación en ciencias sociales (pp. 427-463). Madrid, España: Síntesis.

Ahern, L., Bortree, D. \& Smith, A. (2013). Key trends in environmental advertising across 30 years in National Geographic magazine. Public Understanding of Science, 22(4), 479494.

Banerjee, S., Gulas, C. \& Iyer, E. (1995). Shades of green: A multidimensional analysis of environmental advertising. Journal of Advertising, 24(2), 21-31.

Barthes, R. (1986). Retórica de la imagen. En Autor, Lo obvio y lo obtuso (pp. 29-47). Barcelona, España: Paidós.

Batey, M. (2013). El significado de la marca: cómo y por qué ponemos sentido a productos y servicios. Buenos Aires, Argentina: Granica.

Bernárdez, A. (ed.) (2007). Mujeres inmigrantes en España: Representaciones en la información y percepción social. Madrid, España: Fragua.

Bryman, A. (2012). Social research methods (4a ed.). Oxford, Reino Unido: Oxford University.

Carlson, L., Grove, S. \& Kangun, N. (1993). A content analysis of environmental advertising claims: A matrix method approach. Journal of Advertising, 22(3), 27-39.

Coca-Cola European Partners (2014). Coca-Cola Enterprises announces the launch of Coca-Cola Life. Recuperado de https://www.cokecce.com/news-and-events/news/coca-colaenterprises-announces-the-launch-of-coca-cola-Life. Consultado el 2 de abril de 2019.

Crass, D., Czarnitzki, D. \& Toole, A.A. (2019). The dynamic relationship between investments in brand equity and firm profitability: Evidence using trademark registrations. International Journal of the Economics of Business, 26(1), 157-176.

Do Paço, A. \& Reis, R. (2012). Factors affecting skepticism toward green advertising. Journal of Advertising, 41(4), 147-155. 
El Dínamo. (2016). El duro revés que Coca Cola no esperaba con su producto «Life» en Chile. Recuperado de https://www.eldinamo.cl/nacional/2016/02/03/coca-cola-Life-bajaproduccion/. Consultado el 2 de abril de 2019.

Expansión. (2017). El refresco sin azúcar Green Cola llega a España tras conquistar el mercado griego. Recuperado de http://www.expansion.com/empresas/distribucion/2017/06/ 14/59414a00ca47419e268b45f2.html. Consultado el 2 de abril de 2019.

Fernández, J.D. (2013). Principios de estrategia publicitaria y gestión de marcas. Nuevas tendencias de brand management. Madrid, España: McGraw-Hill.

Geertz, C. (1990). La interpretación de las culturas. Madrid, España: Gedisa.

Gómez-Lorenzini, P., Vergara, E., Labarca, C. \& Porath, W. (2016). Publicidad chilena en el marco de un proceso de crecimiento económico: Aspectos formales y roles sociales en la publicidad del retail a fines de los '90. Palabra Clave, 19(1), 304-331.

Greimas, A.J. \& Courtés, J. (1990). Semiótica. Diccionario razonado de la teoría del lenguaje. Madrid, España: Gredos.

Han, E. (2015). Coca-Cola accused of 'greenwashing' with launch of sugar-reduced Coke Life. The Sydney Morning Herald. Recuperado de https://www.smh.com.au/ healthcare/cocacola-accused-of-greenwashing-with-launch-of-sugarreduced-cokeLife-20150330-1mas6u.html. Consultado el 2 de abril de 2019.

Heding, T., Knudtzen, C.F. \& Bjerre, M. (2009). Brand management. Research, theory and practice. Nueva York (NY): Routledge.

Hofherr, J. (2014). Is the new Coca-Cola 'Life' healthier than regular Coke? (and will it come to the US?). The Boston Globe Online. Recuperado de https://www.boston. $\mathrm{com} /$ news/business/2014/06/17/is-the-new-coca-cola-Life-healthier-than-regularcoke-and-will-it-come-to-the-us. Consultado el 2 de abril de 2019.

Holt, D. (2004). How brands become icons: The principles of cultural branding. Boston (MA): Harvard Business.

Iyer, E.; Banerjee, S. \& Gulas, C. (1994). An expose on green television ads. Advances in Consumer Research, (21), 292-298.

Jolly, W. (2019). The 6 most effective types of social media advertising in 2019. BigCommerce. Recuperado de https://www.bigcommerce.com/blog/social-media-advertising. Consultado el 2 de julio de 2019.

Kangun, N., Carlson, L. \& Grove, S.J. (1991). Environmental advertising claims: A preliminary investigation. Journal of Public Policy y Marketing, 10(2), 47-58.

Leonidou, L., Leonidou, C., Palihawadana, D. \& Hultman, M. (2011). Evaluating the green advertising practices of international firms: A trend analysis. International Marketing Review, 28(1), 6-33.

Leonidou, L., Leonidou, C. \& Kvasova, O. (2010). Antecedents and outcomes of consumer environmentally friendly attitudes and behavior. Journal of Marketing Management, 26(13-14), 1319-1344.

Mangold, W.G. \& Faulds, D.J. (2009). Social media: The new hybrid element of the promotion mix. Business Horizons, 52(4), 357-365. 
Matthes, J. \& Wonneberger, A. (2014). The skeptical green consumer revisited: Testing the relationship between green consumerism and skepticism toward advertising. Journal of Advertising, 43(2), 115-127.

Mazzuco, J. (2016). Coca-Cola reducirá producción de versión Life ante débiles resultados. El Mercurio. Recuperado de http://www.economiaynegocios.cl/noticias/noticias. asp?id=223395. Consultado el 2 de abril de 2019.

Miller, C. (2009). Sustainable marketing and the green consumer. En E. Parsons \& P. Maclaran (Eds.), Contemporary issues in marketing and consumer behaviour (pp. 141-160). Oxford, Reino Unido: Elsevier.

Neuendorf, K. (2002). The content analysis guidebook. Thousand Oaks (CA): Sage.

Ollé, R. \& Riu, D. (2009). El nuevo brand management: cómo plantar marcas para hacer crecer negocios. Barcelona, España: Gestión 2000.

Ortegón, L. (2014). Gestión de marca. Conceptualización, diseño, registro, construcción y evaluación. Bogotá, Colombia: Politécnico Grancolombiano.

Porath, W., Vergara, E., Gómez-Lorenzini, P. \& Labarca, C. (2017). Cambios en la presentación de los personajes de la publicidad del retail en Chile en un contexto de modificaciones en la lógica de consumo asociadas a transformaciones socioculturales (19972013). Comunicación y Sociedad, (29), 203-225.

Regatto, J. del P. \& Viteri, V.M. (2017). Mecanismos de medición para el comportamiento del consumidor utilizados por agencias de mercado en Ecuador. Caso: ConsumerLab vs Mktrends. Observatorio de la Economía Latinoamericana. Recuperado de http://www. eumed.net/cursecon/ecolat/ec/2017/mktrends.html.

Russell, J.T., Lane, W.R. \& King, K.W. (2005). Kleppner: Publicidad (16a ed.). México, D.F.: Pearson Educación.

Schroeder, J. (2009). The cultural codes of branding. Marketing Theory, 9(1), 123-126.

Shrum, L.J., McCarty, J.A. \& Lowrey, T.M. (1995). Buyer characteristics of the green consumer and their implications for advertising strategy. Journal of Advertising, 24(2), $71-82$.

Spessot, C., Testa, L., Van Kooten, M., Velásquez, M., Villalón, M.A. \& Saravi, A. (2016). La publicidad y los recursos persuasivos emocionales. Construcción de estereotipos emocionales y culturales para la promoción de productos. Ponencia presentada en las II Jornadas Estudiantiles de Investigación en Disciplinas Artísticas y Proyectuales, Facultad de Bellas Artes, Universidad Nacional de La Plata, Argentina.

Truong, A. (2013). Green Coke? In Argentina, Coca-Cola Life features green label, bottle, and ingredients. FastCompany Online. Recuperado de https://www.fastcompany. com/3014667/green-coke-in-argentina-coca-cola-Life-features-green-label-bottleand-ingredients. Consultado el 2 de abril de 2019.

Tucker, E., Rifon, N., Lee, E.M. \& Reece, B. (2012). Consumer receptivity to green ads. A test of green claim types and the role of individual consumer characteristics for green ad response. Journal of Advertising, 41(4), 9-23. 
US Food and Drug Administration (2018). Has Stevia been approved by FDA to be used as a sweetener? Recuperado de https://www.fda.gov/AboutFDA/Transparency/Basics/ ucm194320.htm. Consultado el 2 de abril de 2019.

Valero, M. (2017). Burbujas con stevia: todos los intentos ¿frustrados? por ofrecer refrescos sin azúcar. El Confidencial. Recuperado de https://www.elconfidencial.com/ economia/2017-12-25/stevia-coca-cola-refrescos-azucar-green-cola-pepsi1497594/. Consultado el 2 de abril de 2019.

Vergara, E. (2006). Publicidad: la zanahoria metafisica que mueve al burro o el color de nuestro tiempo. Trípodos, (18), 157-164.

Vergara, E. \& Porath, W. (2017). Representaciones de la mujer en la gráfica chilena. Del protagonismo político a la subjetividad del consumo. Gráfica, 5(10), 139-144.

Wallop, H. (2014). Taste test: does Coca Cola Life taste better than regular Coke? The Telegraph. Recuperado de https://www.telegraph.co.uk/finance/newsbysector/ retailandconsumer/11050410/Taste-Test-does-Coca-Cola-Life-taste-better-thanregular-Coke.html. Consultado el 2 de abril de 2019.

Williamson, J. (1978). Decoding advertisements. Londres, Reino Unido: Marion Boyars.

Wilson, M. (2013). Coca-Cola debuts 'Life' brand, highlights deadliness of regular Coke. FastCompany Online. Recuperado de https://www.fastcompany.com/1673295/cocacola-debuts-Life-brand-highlights-deadliness-of-regular-coke. Consultado el 2 de abril de 2019.

Zinkhan, G.M. \& Carlson, L. (1995). Green advertising and the reluctant consumer. Journal of Advertising, 24(2), 1-6. 